\title{
Mate choice and the operational sex ratio: an experimental test with robotic crabs
}

\author{
Hayes, Catherine L ; Callander, Sophia ; Booksmythe, Isobel ; Jennions, Michael D ; Backwell, Patricia R Y
}

\begin{abstract}
The operational sex ratio (OSR: sexually active males: receptive females) predicts the intensity of competition for mates. It is less clear, however, under what circumstances, the OSR predicts the strength of sexual selection - that is, the extent to which variation in mating success is attributable to traits that increase the bearer's attractiveness and/or fighting ability. To establish causality, experiments that manipulate the OSR are required. Furthermore, if it is possible to control for any OSR-dependent changes in the chosen sex (e.g. changes in male courtship), we can directly test whether the OSR affects the behaviour of the choosing sex (e.g. female choice decisions). We conducted female mate choice experiments in the field using robotic models of male fiddler crabs (Uca mjoebergi). We used a novel design with two females tested sequentially per trial. As in nature, the choice of the first female to mate therefore affected the mates available to the next female. In general, we detected significant sexual selection due to female choice for 'males' with larger claws. Importantly, the strength of sexual selection did not vary across five different OSR/density treatments. However, as the OSR decreased (hence the number of available males declined), females chose the 'males' with the largest claws available significantly more often than expected by chance. Possible reasons for this mismatch between the expected and observed effects of the OSR on the strength of sexual selection are discussed.
\end{abstract}

DOI: https://doi.org/10.1111/jeb.12884

Posted at the Zurich Open Repository and Archive, University of Zurich

ZORA URL: https://doi.org/10.5167/uzh-124248

Journal Article

Accepted Version

Originally published at:

Hayes, Catherine L; Callander, Sophia; Booksmythe, Isobel; Jennions, Michael D; Backwell, Patricia R Y (2016). Mate choice and the operational sex ratio: an experimental test with robotic crabs. Journal of Evolutionary Biology, 29(7):1455-1461.

DOI: https://doi.org/10.1111/jeb.12884 


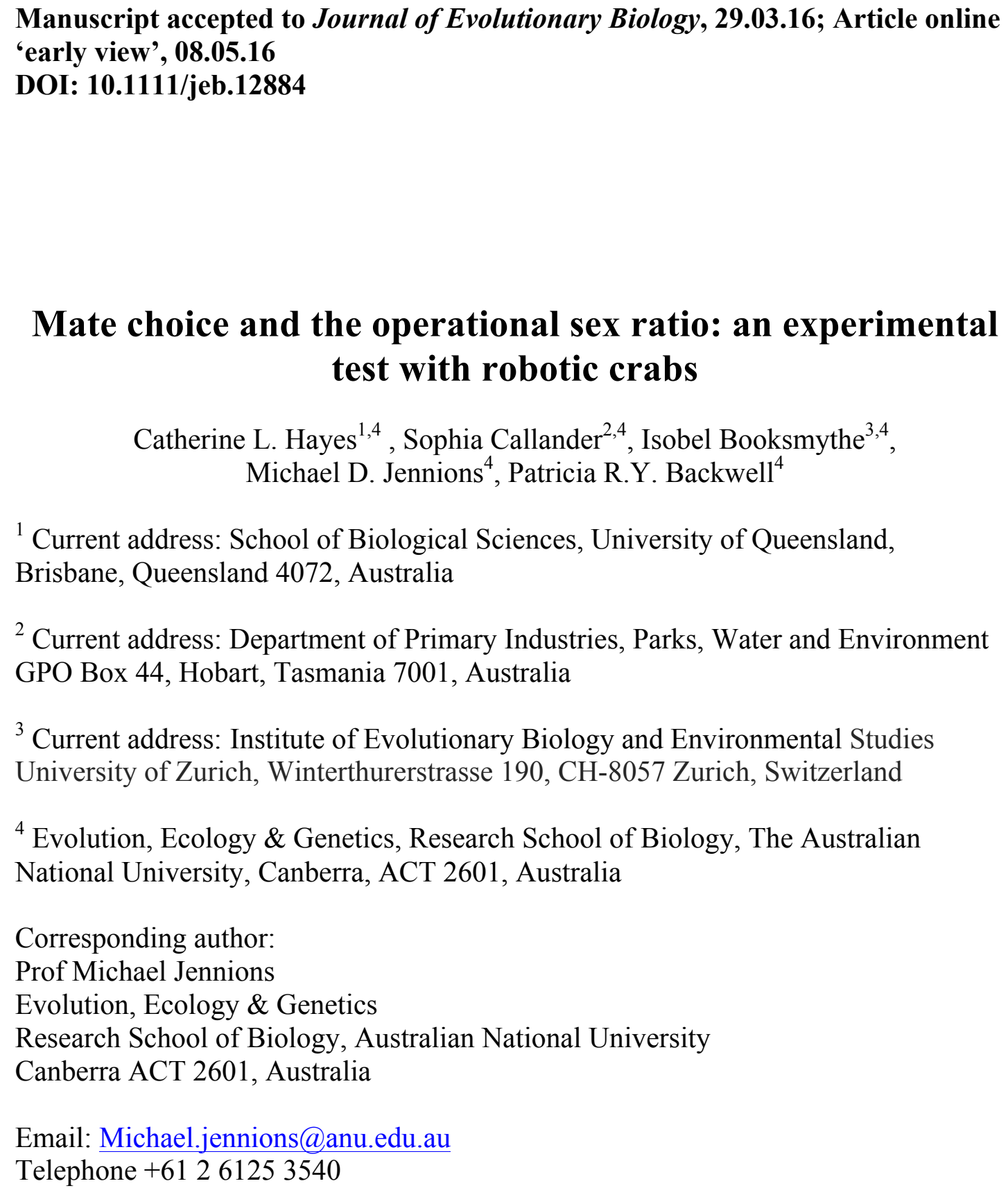

\section{Mate choice and the operational sex ratio: an experimental test with robotic crabs}

Title: Mate choice and the operational sex ratio: an experimental test 
41 Abstract: The operational sex ratio (OSR) (sexually active males: receptive females) predicts the intensity of competition for mates. It is less clear, however, under what circumstances the OSR predicts the strength of sexual selection - that is, the extent to which variation in mating success is attributable to traits that increase the bearer's attractiveness and/or fighting ability. To establish causality experiments are required that manipulate OSR. Furthermore, if it is possible to control for any OSR-dependent changes in the chosen sex (e.g. changes in male courtship), we can directly test whether the OSR affects the behaviour of the choosing sex (e.g. female choice decisions). We conducted female mate choice experiments in the field using robotic models of male fiddler crabs (Uca mjoebergi). We used a novel design with two

51 females tested sequentially per trial. As in nature, the choice of the first female to mate therefore affected the mates available to the next female. In general we detected significant sexual selection due to female choice for 'males' with larger claws. Importantly, the strength of sexual selection did not vary across five different OSR/density treatments. However, as the OSR decreased (hence the number of available males declined), females chose the 'males' with the largest claws available significantly more often than expected by chance. Possible reasons for this mismatch between the expected and observed effects of the OSR on the strength of sexual selection are discussed.

60

61 Keywords: density, female choice, mate sampling, mating preferences 
It is well established that sexual selection has led to the evolution of costly male traits that are advantageous during male-male contests, or when competing to attract females (Andersson, 1994). It is less clear what demographic or ecological factors generate variation among species or populations in these secondary sexual traits (Cornwallis \& Uller, 2010). One parameter traditionally used to predict the strength of sexual selection is the operational sex ratio (OSR: ratio of sexually active males to receptive females) (Emlen \& Oring, 1977; for a review of sexual selection indices see Henshaw et al., 2016). By definition the intensity of competition for mates is stronger when the OSR is more biased. Greater mating competition need not, however, equate to stronger sexual selection. This is because sexual selection is measured as the response to competition (i.e. the extent to which variation in mating success among individuals is due to differences in the expression of sexual traits) (Shuster, 2009; Klug et al., 2010; Jennions et al., 2012). In some cases, it is more profitable to invest in other fitness-enhancing traits when the intensity of mating competition increases (e.g. parental care; Kokko \& Jennions, 2008). As the OSR becomes increasingly male-biased, variation in male mating success attributed to individual differences in sexually selected traits might therefore even decrease. sexual selection on focal traits cannot be derived from first principles. First, there is an imperfect correlation between the intensity of sexual competition (i.e. OSR) and a sexually selected response (e.g. greater investment in ornaments or weaponry) if there are also ways to increase fitness via naturally selected traits (e.g. parental care, 
defence against parasitism) (reviews: Kokko \& Jennions, 2008; Jones, 2009). Second, proximate factors that affect the intensity of sexual selection on different traits can change with the OSR (Shuster, 2009; Klug et al., 2010). For example, controlling for density, when the OSR is more male-biased the encounter rates between males will increase. Numerous aggressive interactions might make harem defence less economically viable in some species (Emlen \& Oring, 1977; Klemme et al., 2007). This could reduce sexual selection on weapons, but might increase sexual selection on other sexually selected traits (e.g. testes size if females are then more inclined to mate multiply). Third, although it is counter-intuitive, all else being equal, female mate sampling tactics that involve assessment of a random subset of males yield the same strength of sexual selection on preferred male traits regardless of the OSR (Klug et $a l ., 2010)$. Fourth, all else is not necessarily equal, however, as greater choice can reduce the ability of choosy individuals to identify preferred items, including mates (e.g. consumers/shoppers: Lenton \& Francesconi, 2011; but see Scheibehenne et al., 2010; mate-searching animals: Hutchinson, 2005; Alem et al., 2015). The density of one sex and the OSR are related, and they covary perfectly if the population density is constant. Consequently, with a more biased OSR, there are often more mates available for the rarer sex to choose from at any moment.

\section{There is no formal theoretical link between the OSR and the strength of sexual} selection on focal male traits, so the true relationship has to be determined empirically (Klug et al., 2010). Comparative analyses suggest that male-biased sex ratios are generally associated with greater male ornamentation and/or weaponry, but the OSR covaries with other factors, notably the adult sex ratio, so it is problematic to assign a causal role to the OSR (Fromhage \& Jennions, submitted). Experimental studies that 
114 manipulate the OSR to determine its causal effects yield contrasting results. For

115 example, as expected, sexual selection on male body size increased as the OSR was

116 made more male-biased in two-spotted gobbies, Gobiusculus flavescens (Wacker et

117 al., 2013). There was, however, no relationship between the OSR and the strength of

118 sexual selection on attractive male traits in guppies, Poecilia reticulata (Head et al.,

119 2008) or mosquitofish, Gambusia holbrooki (Head et al. submitted); and there was

120 weaker sexual selection on male body size in bank voles Clethrionomys glareolus

121 (Klemme et al., 2007) and common lizards Lacerta vivipara (Fitze \& Le Galliard,

122 2008) when the OSR was made more male-biased.

123

124 Experiments manipulating the OSR are needed to determine whether general trends can be identified. For example, is the OSR a better predictor of sexual selection in some types of mating systems and/or taxa than others? Such OSR manipulation experiments, while straightforward, are surprisingly few in number. Even more rare are studies where OSR-dependent behavioural changes in one sex are controlled for,

129 so that the direct effect of the OSR on the other sex can be calculated. For example, if 130 female choice based on male courtship varies with the OSR, is this due to OSR-

131 dependent changes in how males court, or in how females evaluate males? To tease 132 sex-specific effects apart necessitates experiments that use artificial stimuli, such as 133 acoustic playbacks or robots, so that 'male' behaviour is unaffected by the OSR.

135 Here, we examine how the OSR affects female choice for male claw size in fiddler 136 crabs. We tested females with custom-built robots 'males' that we have successfully 137 used in many previous studies (e.g. Reaney, 2009; Kahn et al., 2013). To avoid any 138 confusion about our non-standard experimental design (two females per trial, see 
139 Methods), we note three points. First, the OSR changes constantly but, to be a useful

140 predictive tool, it should be measured at a biologically appropriate scale. For example,

141100 calling male frogs and 20 gravid females might be at a pond over a night. Most

142 researchers would report this as a 5:1 OSR (e.g. Ryan, 1981). Strictly speaking,

143 however, for the first female the OSR was 100:1 and for the last female it was $81: 1$

144 (male frogs rarely return to the mating pool on the night that they mate). In general,

145 most researchers are interested in this type of 'population level' OSR. Unless

146 otherwise stated we follow this convention in our study. Specifically, we refer to the

147 OSR at the start of an experimental trial rather than that experienced by successive

148 females within a trial. It is less common to refer to the OSR experienced by an

149 individual female. This is probably because the 'female-level' OSR is synonymous

150 with how many sexually receptive males a female encounters. In such case, rather

151 than refer to the OSR, we simply refer to the number of males available as mates.

153 Second, most mate choice experiments test a single female per scenario so that each

154 female chooses from the same set of stimuli. The disadvantage, hinted at in the frog

155 scenario, is that this design ignores changes in mate availability that will arise when

156 females sequentially arrive to mate. Biologically realistic mate choice experiments

157 could include the possibility that several females chose from the same set of males but

158 that earlier choices constrain future options. Third, as noted earlier, the population

159 level OSR and the total number of available males ('male density') tend to be

160 correlated in the field. Although density and the population level OSR can be teased

161 apart in experiments (Kokko \& Rankin, 2006; Wacker et al., 2013), this does not

162 negate the fact that a more male-biased population level OSR can often equally well

163 be described as a case where females choose among a greater number of males. 
164 Researchers interchangeably describe this as the effect of either greater choice of

165 mates (standard terminology for those interested in proximate sensory mechanisms) or

166 of the OSR (standard terminology for those interested in how sex ratios affect

167 selection gradients) on female mating preferences.

168

METHODS

170

171 We studied Uca mjoebergi at East Point Reserve in Darwin, Australia in October-

172 December 2010. Both sexes defend burrows on inter-tidal mudflats. During the five-

173 day mating period that occurs each semi-lunar tidal cycle, mate-searching females

174 leave their burrow, move across the mudflat and sequentially encounter clusters of 2-

17510 males. This is the main level at which mate choice decisions occur. The population

176 level OSR is always male-biased. Males court females by vigorously waving their

177 enlarged major claw. In the field, females more often approach larger-clawed males

178 (Reaney \& Backwell, 2007) and males that wave earlier (i.e. produce 'leading'

179 waves) (Reaney et al., 2008). After choosing a male the female enters his burrow to

180 inspect it. Burrow features then influence whether she stays and breeds, or continues

181 mate searching (Reaney \& Backwell, 2007). Sexual selection presumably favours

182 males with large claws that increase the likelihood that a female inspects his burrow.

183 Male claw size in the population at the time of our study ranged from 4.6 to $25.4 \mathrm{~mm}$

184 (mean \pm SD: $14.97 \pm 4.29 \mathrm{~mm} ; n=222$; based on transects). This is consistent with

185 three recent estimates from the same study area [14.84 $\mathrm{mm}(n=82), 14.95 \mathrm{~mm}(n=$

186 199) and $14.9 \mathrm{~mm}(n=177)$; Clark \& Backwell 2015). Even though males within this

187 size range all produce courtship waves, it is possible that the smaller males have

188 burrows that are too narrow for females. A better estimate of the size range of males 
available as mates is obtained by restricting the dataset to males whose burrows are inspected by females. Three recent estimates from the field at the same site for the mean \pm SD claw size of these males are $14.84 \pm 1.63(n=37), 16.05 \pm 2.29(n=135)$ and $16.60 \pm 2.92 \mathrm{~mm}(n=57)($ data from Clark \& Backwell 2015).

We ran mate choice experiments where we sequentially presented two test females with 3,5 or 7 robotic 'males' (i.e. the population level OSR over the trial was 7:2, 5:2 or 3:2, and the OSR for individual females ranged from $7: 1$ to $2: 1$ ). Each robot consists of a painted cast of a claw attached to a motorised metal arm that mimics courtship waving (details in Reaney et al., 2008). Robots waved in synchrony so that leadership did not affect female choice. The test arena was an area of mudflat from which we removed all resident crabs. We used mate-sampling females caught in situ who were measured (carapace width $\pm 0.1 \mathrm{~mm}$ ) and held in individual containers until tested $(<30$ mins). We placed the first female under a clear plastic cup at a point equidistant from all robots $(20 \mathrm{~cm})$. After the female settled we raised the cup and scored a choice decision if she moved to $<2 \mathrm{~cm}$ of a 'male'. A female was discarded and replaced with another if she ran immediately after release, or did not choose within 3 minutes $(n=47)$. This criterion for choice produces highly repeatable results in the many studies we have conducted (e.g. Reaney, 2009; Kahn et al., 2013). The results from female choice trials using robotic crabs have largely been corroborated by field studies of correlates of male mating success (e.g. Reaney \& Backwell, 2007; Clark \& Backwell 2015). Given the large numbers of mate-searching females, and that females mate on the day that they begin to search (Clark \& Backwell 2015), it is highly improbable that we tested a female more than once. The first female saw all the 'males' wave before she was released. Her chosen 'male' was then removed and the 
214 second female chose from the remaining 'males'. This mimics the natural situation. In

215 thousands of hours of field observations we have almost never seen two females

216 simultaneously approach a cluster of males - rather, females sequentially approach

217 sets of males. Mate choice by an earlier female removes the chosen male from the set

218 of potential mates of the next female because a male remains underground once a

219 female has chosen to stay in his burrow. Ideally we would have collected data blind to

220 the OSR and male claw size, but this was not possible (see Holman et al. 2015).

221 Female choice of a given 'male' is, however, clear-cut based on her behaviour, and

222 we had no a priori expectations as to how the OSR would affect female choice.

224 We used three high-density treatments where the spacing between 'males' was

225 constant $(5 \mathrm{~cm})$ to test if the OSR affects the strength of sexual selection: 7:2, 5:2 and

226 3:2 (Fig 1a). Our design reflects the clusters of waving males encountered by a mate-

227 searching female. The smallest, median and largest claw sizes we used were always

228 the same (Table 1). The mean 'male' claw size $(18.2 \mathrm{~mm})$ was larger than the mean

229 for males whose burrows are inspected (16.0 mm; Clark \& Backwell, 2015): 10 of

230 the 15 'males' had claws greater than $16.0 \mathrm{~mm}$. This makes tests for a directional

231 mating preference conservative, although only moderately so given that naturally 7 of

23215 males are larger than average (assuming the median and the mean are the same).

233 The chosen size range was necessary to ensure the tested claws spanned the natural

234 size range, but were evenly distributed in size while keeping the mean value constant.

235 The position of robots with different sized claws was randomised. To test for an

236 effect of male density independent of the OSR (e.g. Head et al., 2008, Wacker et al.,

237 2013) we re-ran the 5:2 and 3:2 OSR treatments at lower densities, adjusting the

238 spacing between robots to cover the same area as the 7:2 treatment (Fig. 1b). We ran 
23940 trials $(=80$ females $)$ per treatment $(n=400$ choice trials $)$. Each female was used in

240 one trial/treatment. We randomised the order of the treatments during each day of

241 testing.

243 To quantify sexual selection we calculated the selection differential $(s)$ as the mean

244 size of the chosen claws minus the original mean claw size. Unless otherwise stated

245 this is based on the mean value of the two females. We ran $t$-tests to determine

246 whether $s$ differed from zero, and an ANOVA to test whether $s$ differed among the

247 OSR treatments with even 'male' spacing. We used a general linear model to test

248 whether density (high/low) and its interaction with the OSR (3:2 or 5:2) affected $s$.

249 There was no effect of day of cycle (relative to spring tide) on $s$ (or the difference

250 between the observed and maximum possible value of $s$ ), nor did day of cycle interact

251 with OSR treatment (all $p>0.254)$.

253 When the OSR is more male-biased, the mean size of the two largest males is greater

254 (Table 1). To determine whether female choice is more error prone when the OSR is 255 more male-biased it is tempting to test whether the difference between the mean size

256 of the chosen males and the maximum mean possible increases with the OSR. This is, 257 however, a problematic approach as the probability that by chance alone the two

258 largest males are chosen is higher when the OSR is less male biased (i.e. there are

259 fewer males). The null prediction is that, by chance, the two largest males are chosen

2601 in 3 times when the OSR is 3:2, 1 in 10 times when the OSR is 5:2 and 1 in 21 times

261 when the OSR is 7:2. We therefore tested whether the observed number of times the

262 two largest 'males' were chosen was significantly greater than expected using

263 separate one-tailed binomial tests. To indirectly compare female error rates among the 
high density OSR treatments we ran an ANOVA to test whether the claw size chosen by the first female per trial differed (this is equivalent to asking whether s differed because the mean claw size is identical for all three OSRs).

Finally, we compared $s$ between the first and second female ( $s$ for the second female was based on the mean size of the remaining available claws) with a paired $t$-test. We tested whether there was an effect of the OSR treatment on the difference in $s$ between the first and second female using separate ANOVAs for high and low density tests. We also calculated the correlation between female size and chosen claw size for each treatment. We used the first female per trial to ensure comparable male availability. All tests were two-tailed $(\alpha=0.05)$ and run in SPSS 19.0.

\section{RESULTS}

In the high-density treatments the selection differential was significantly greater than zero at all three OSRs. Females prefer larger claws: 7:2 $\left(t_{39}=2.968, p=0.005\right), 5: 2$ $\left(t_{39}=2.507, p=0.016\right)$ and $3: 2\left(t_{39}=3.798, p<0.001\right)($ Table 1$)$. The strength of sexual selection $(s)$ did not depend on the $\operatorname{OSR}\left(F_{2,117}=0.242, p=0.785 ; 7: 2=\right.$ $1.187 \mathrm{~mm} ; 5: 2=0.98 \mathrm{~mm} ; 3: 2=1.35 \mathrm{~mm})$. Females in the $3: 2$ treatment more often chose the two largest available claws than did females in the $5: 2$ or 7:2 treatments. The frequency of trials in which the two largest males were chosen was significantly greater than expected by chance for the $3: 2$ treatment ( 24 of 40 trials, $p=0.001$ ), but not for the 5:2 (4 of 40 trials) or 7:2 treatments ( 2 of 40 trials) (both $p>0.50$ ). There was, however, no evidence that the first female more often selected the largest claw at a less male-biased OSR as the mean claw size in first trials did not differ across 
treatments $\left(F_{2,117}=0.482, p=0.62 ;\right.$ mean claw size: $3: 2=18.35 \mathrm{~mm}, ; 5: 2=19.08 \mathrm{~mm}$, 7:2 $=19.19 \mathrm{~mm})$. The greater than expected number of choices of the two largest males in the 3:2 treatment must therefore be driven by the choice of the second

292 female.

294 In the low-density treatments, although females still tended to choose larger claws, sexual selection for larger claws was not significant at either a 3:2 $\left(t_{39}=1.312, p=\right.$ $0.197)$ or $5: 2$ OSR $\left(t_{39}=1.936, p=0.06\right)$. The frequency of trials in which the two

297 largest males were chosen was, however, not significantly greater than expected by 298 chance in either the $3: 2$ treatment (18 of 40 trials, $p=0.083)$, or the 5:2 treatment ( 7 299 of 40 trials, $p=0.10$ ). There was no evidence that the first female more often selected 300 the largest claw at a less male-biased OSR, as the mean claw did not differ $\left(F_{1,78}=\right.$ $0.860, p=0.357 ; 3: 2=19.55 \mathrm{~mm}, ; 5: 2=18.55 \mathrm{~mm})$.

303 When analysing results from the two densities together, there was no significant effect 304 of density on the strength of sexual selection $(s)\left(F_{1,156}=2.076, p=0.152\right)$, nor was 305 there a density by OSR interaction $\left(F_{1,156}=0.548, p=0.460\right)$ or a main effect of OSR $306\left(F_{1,156}=0.063, p=0.802\right)($ Mean s was 1.35 and $0.525 \mathrm{~mm}$ at high and low densities 307 for the 3:2 OSR, and 0.975 and $0.710 \mathrm{~mm}$ at high and low densities for the 5:2 OSR).

309 Combining all available trials there was no evidence that the selection differential of 310 the second female was stronger than that of the first female $\left(t_{199}=1.533, p=0.127\right.$;

311 first $=0.756 \mathrm{~mm}$, second $=1.389 \mathrm{~mm}$ ). There was no effect of the OSR on the 312 magnitude of the difference in the selection differential between the first and second 313 female (low-density: $F_{2,117}=1.779, p=0.173$; high-density: $F_{1,78}=1.653, p=0.202$; 
314 the direction of the effect was first $s<\operatorname{second} s$ at both densities).

316 Finally, female size was uncorrelated with chosen claw size in all five treatments $(r=$

$3170.173,-0.058,0.157,0.053,-0.027$, all $p>0.285)$. The mean correlation was $r=$

$3180.060\left(t_{4}=1.275, p=0.271\right)$. There was no evidence that larger claws additionally

319 enhance male fitness by preferentially attracting larger, more fecund, females.

\section{DISCUSSION}

Female Uca mjoebergi preferred to visit larger-clawed robotic 'males' in three of our

324 five OSR/density treatments, and there was a marginally non-significant preference in one treatment $(p=0.06)$. These results corroborate findings from comparable mate choice experiments with robotic crabs (e.g. Reaney, 2009; Kahn et al., 2013). They also agree with our estimates of male mating success in the field (Reaney \& Backwell, 2007). Our mate choice experiments were conservative with respect to

329 female choice for larger males as the mean test claw size $(18.2 \mathrm{~mm})$ was larger than 330 the population mean $(14.9 \mathrm{~mm})$. The disparity was smaller when using the estimated 331 mean of $16.0 \mathrm{~mm}$ based on males naturally visited by females (from Clark \& Backwell 332 2015). Ten of the 15 'males' available across the three OSR treatments were larger 333 than this mean. The strong directional female mating preference for larger claws that 334 we still observed can therefore partly explain why, like all fiddler crabs, male $U$. mjoebergi have a greatly enlarged major claw.

337 Although directional selection on claw size was not statistically significant in all 338 treatments, the strength of sexual selection due to female choice did not depend on the 
OSR. More specifically, sexual selection was not stronger when the OSR was more

340 male-biased. In general, this positive trend is widely predicted because the mean size

341 of the two largest claws was bigger when the OSR was more male biased (Table 1). If

342 each female chose the largest available claw with the same propensity irrespective of

343 the OSR, the strength of sexual selection should have increased with a more male-

344 biased OSR. (There is no confounding effect of mate-sampling tactics affecting the

345 mean size of available males [for examples see Klug et al., 2010] because females had

346 simultaneous access to all available 'males'). Instead, we found that the females'

347 propensity to choose the two largest claws available decreased as the OSR became

348 more male-biased. Specifically, females chose the two largest claws significantly

349 more often than expected by chance only in the least male-biased OSR (3:2, at high

350 density). The absence of a relationship between the OSR and strength of sexual

351 selection in U. mjoebergi is a reminder that the OSR is an imperfect predictor (Jones,

352 2009; Shuster, 2009). Indeed, a recent simulation study of various mating systems that

353 tested a range of proposed indices of sexual selection, showed that the OSR tended to

354 be a consistently poor predictor of sexual selection on a focal trait (Henshaw et al., 355 2016).

357 There are several possible reasons why the OSR did not predict the strength of sexual 358 selection driven by female choice for larger claws in U. mjoebergi. To start though, 359 we can eliminate any role of OSR-dependent changes in male behaviour (review:

360 Weir et al., 2011) that might affect the attractiveness of a larger claw to females. This

361 is because we tested females using robotic 'males' with constant wave rates. An OSR-

362 mediated change in non-focal traits is potentially important in the field because the

363 correlation between claw size and wave rate, which are both traits affecting male 
attractiveness, varies over the mating period in U. mjoebergi (Kahn et al., 2013).

366 We suggest that the OSR affected the strength of sexual selection due to psycho-

367 sensory processes that altered a female's ability to discriminate between males and

368 chose the largest available. The greater potential for stronger sexual selection with a

369 more male-biased OSR (because the two largest available claws have a greater mean

370 size) is then negated by increased mistakes by females so that there is no net effect of

371 the OSR on the strength of sexual selection. There are two factors likely to increase

372 female error rates in identifying the largest claw. First, the size difference between

373 adjacent sized 'males' declined from $6 \mathrm{~mm}$ to $2 \mathrm{~mm}$ as the OSR went from 3:2 to 7:2

374 (Table 1). This is not an experimental flaw: it is inevitable that the size difference

375 between adjacent sized 'males' decreases when more are present. Discrimination

376 between similar sized objects is always more difficult (citations in Abbas et al., 2013).

377 It is worth noting that the mean claw size chosen by the first female did not differ

378 among the OSR treatments. However, the proportion of tests in which the two largest

379 males were selected was greater than expected by chance was only significantly for

380 the 3:2 OSR treatment. This suggests that it is the choice of the second female that

381 drives this result, implying that when there are only two males to chose between in the

$3823: 2$ OSR treatment females are more likely to select the largest available male than

383 when there are four or six available males as in the 5:2 and 7:2 OSR treatments.

385 Second, the number of 'males' present increased with the OSR, again reflecting the

386 situation in the field. There is some evidence that choice of a preferred item becomes

387 more difficult when there are more items to choose from (review: Hutchinson, 2005).

388 Together this implies that the number of 'males' and/or size-differences between 
'males' that both covaried with the OSR increased the error rate of choosy females.

390 Similar relationships seem likely to occur in many taxa where the biologically

391 relevant OSR largely depends on the number of males present on a breeding/mating

392 site while individual females are sampling males. Arguing against a role for the

393 number of males and/or size-differences between males is that there was no

394 significant increase in the selection differential of the second female relative to that of

395 the first female. This occurred even though the second female saw one fewer 'male',

396 and, on average, there was a greater size difference between the largest and next

397 largest 'male'. It is worth noting, however, that we might have reported a significant

398 effect if we had used a different distribution of claw sizes so that there were fewer

399 'males' with above average sized claws (relative to the natural mean). In such a case,

400 the choice of a large 'male' by the first female would have increased the proportion of

401 below average 'males' so that females might have become less choosy. More

402 convincingly, in previous two-choice experiments the only treatment in which

403 females did not choose the bigger claw was when they differed in size by just $2 \mathrm{~mm}$

404 (Reaney 2009).

405

406 To test whether the number of 'males' per se has a direct effect on female choice it is

407 necessary first to control for the size differences between 'males', and vary the

408 number of 'males' (e.g. double the number of 'males' in the 3:2 OSR treatment and

409 compare the 3:2 and 6:2 treatments). It should be noted, however, that in the

410 proposed, as well as the current, experiment the variance in male claw size is greater

411 when the OSR is less male biased. This is a constraint of the design given the decision

412 to have the same range in claw size. In a natural setting the number of males that an

413 individual female encounters might not show the same relationship with variation in 
414 male claw size. Finally, it is possible that the OSR directly changes female mating

415 preferences. Females might have a weaker preference for larger males when, say,

416 more males are present although there is no obvious adaptive advantage to such a shift

417 in mating preference.

419 In conclusion, the proximate mechanisms underlying consistent sexual selection for 420 larger claw size independent of the OSR in U. mjoebergi are unknown. This does not, 421 however, negate our key finding sexual selection theory. Despite the OSR covarying 422 with the opportunity for sexual selection (i.e. maximum selection differential) sexual 423 selection on male claw size did not increase as the OSR became more male-biased.

\section{DATA ACCESSIBILITY}

426 Data for this study is available from the DRYAD repository doi:10.5061/dryad.5qb78

\section{REFERENCES}

Abbas, S.S., Dijkstra, T.M.H. \& Heskes, T. 2013. A direct comparison of visual discrimination of shape and size on a large range of aspect ratios. Vision Research 91: 84-92.

Alem, S., Clanet, C., Party, V., Dixsaut, A. \& Greenfield, M.D. 2015. What determines lek size? Cognitive constraints and per capita attraction of females

436 Andersson, M.B. 1994. Sexual Selection. Princeton University Press, Princeton, NJ.

437 Clark, H.L. \& Backwell, P.R.Y. 2015. Temporal and spatial variation in female 438 mating preferences in a fiddler crab. Behav. Ecol. Sociobiol. 69: 1779-1784. 
439 Cornwallis, C.K. \& Uller, T. 2010. Towards an evolutionary ecology of sexual traits.

$440 \quad$ Trends Ecol. Evol. 25: 145-152.

441 Emlen, S.T. \& Oring, L.W. 1977. Ecology, sexual selection, and the evolution of 442 mating systems. Science 197: 215-223.

443 Fitze, P.S. \& Le Galliard, J.F. 2008. Operational sex ratio, sexual conflict and the $444 \quad$ intensity of sexual selection. Ecol. Lett. 11: 432-439.

445 Head, M.L., Lindholm, A.K. \& Brooks, R. 2008. Operational sex ratio and density do 446 not affect directional selection on male sexual ornaments and behavior. Evolution $447 \quad 62: 135-144$.

448 Henshaw, J., Kahn, A.T. \& Fritzsche, K. 2016. A rigorous comparison of sexual 449 selection indices via simulations of diverse mating systems. Proc Natl. Acad. Sci. U.S.A. 113: E300-E308.

451 Holman, L., Head, M.L., Lanfear, R. \& Jennions, M.D. 2015. Evidence of 452 experimental bias in the life sciences: why we need blind data recording. PLoS $453 \quad$ Biology 13: e1002190.

454 Hutchinson, J.M.C. 2005. Is more choice always desirable? Evidence and arguments 455 from leks, food selection, and environmental enrichment. Biol. Rev. 80: 73-92. 456 Jennions, M.D., Kokko, H. \& Klug, H. 2012. The opportunity to be misled in studies

Jones, A.G. 2009. On the opportunity for sexual selection, the Bateman gradient and of sexual selection. J Evol. Biol. 25: 591-598.

460 Kahn, A.T., Dolstra, T., Jennions, M.D. \& Backwell, P.R.Y. 2013. Strategic male

461 courtship effort varies in concert with adaptive shifts in female mating 462 preferences. Behav. Ecol. 24: 906-913

463 Klemme, I., Ylonen, H. \& Eccard, J.A. 2007. Reproductive success of male bank 
voles (Clethrionomys glareolus): the effect of operational sex ratio and body size. Behav. Ecol. Sociobiol. 61: 1911-1918.

466

Klug, H., Heuschele, J., Jennions, M.D. \& Kokko, H. 2010. The mismeasurement of sexual selection. J. Evol. Biol. 23: 447-462.

Kokko, H. \& Jennions, M.D. 2008. Parental investment, sexual selection and sex ratios. J. Evol. Biol. 21: 919-948.

Kokko, H. \& Rankin, D.J. 2006. Lonely hearts or sex in the city? Density-dependent effects in mating systems. Phil. Trans. Roy. Soc. B. 361: 319-334.

Lenton, A.P. \& Francesconi, M. 2011. Too much of a good thing? Variety is confusing in mate choice. Biol. Letts. 7: 528-531.

Reaney, L.T. \& Backwell, P.R.Y. 2007. Temporal constraints and female preference for burrow width in the fiddler crab, Uca mjoebergi. Behav. Ecol. Sociobiol. 61: $1515-1521$.

Reaney, L.T., Sims, R.A., Sims, S.W., Jennions, M.D. \& Backwell, P.R.Y. 2008. Experiments with robots explain synchronized courtship in fiddler crabs. Curr. Biol. 18: R62-R63.

Reaney, L.T. 2009. Female preference for male phenotypic traits in a fiddler crab: do females use absolute or comparative evaluation? Anim. Behav. 77: 139-143.

Ryan, M.J. 1981. The Tungara Frog. University of Chicago Press, Chicago.

Scheibehenne, B., Greifender, R. \& Todd, P.M. 2010. Can there ever be too many options? A meta-analytic review of choice overload. J Consumer Research 37: $409-425$.

Shuster, S.M. 2009. Sexual selection and mating systems. Proc. Natl. Acad. Sci. U.S.A. 106: 10009-10016.

Wacker, S., Mobley, K., Forsgren, E., Myhre, L.C., de Jong, K. \& Amundsen, T. 


\begin{tabular}{lcc}
\hline Treatment & Chosen mean \pm SD & Maximum \\
& $($ all $n=40$ trials $)$ & possible mean
\end{tabular}

2013. Operational sex ratio but not density affects sexual selection in a fish. Evolution 67: 1937-1949.

Weir, L.K., Grant, J.W.A. \& Hutchings, J.A. 2011. The influence of operational sex ratio on the intensity of competition for mates. Amer. Nat. 177: 167-176.

Table 1. Available, observed mean of chosen males, and maximum possible mean claw length (i.e. largest two males) (in $\mathrm{mm}$ ) for the five OSR-density treatments.

\section{OSR Male claw length Mean High density Low density}

$\begin{array}{lllll}7: 2 & 12.2,14.1,16.2,18.2, & 18.2 & 19.34 \pm 2.53 & 23.15\end{array}$

$20.2,22.1,24.2$

$5: 2 \quad 12.2,15.2,18.2$

$18.2 \quad 19.16 \pm 2.46 \quad 18.89 \pm 2.32$

$21.1,24.2$

$3: 2 \quad 12.2,18.2,24.2$

$18.2 \quad 19.55 \pm 2.25 \quad 18.73 \pm 2.53$

21.2 
503 Figure 1. Diagram of the three OSR treatments: (a) even male spacing (high density) 504 and (b) spacing such that the same area is covered in all three OSR treatments. Claws: 505 robotic 'males'; crab: female release point.

506
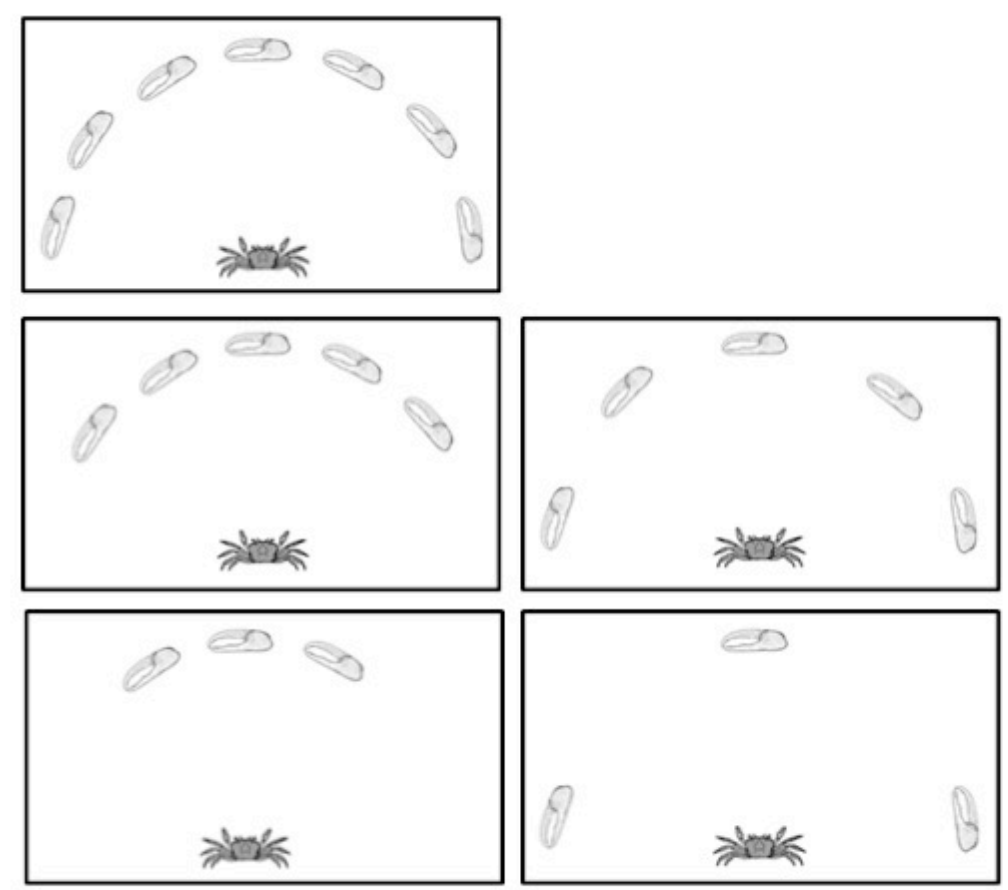

a

b

507 